Article

\title{
Association of Candidate Gene Polymorphism with Metabolic Syndrome among Mongolian Subjects: A Case-Control Study
}

\author{
Ariunbold Chuluun-Erdene ${ }^{1}{ }^{\circledR}$, Orgil Sengeragchaa ${ }^{1}$, Tsend-Ayush Altangerel ${ }^{1}$, \\ Purevjal Sanjmyatav ${ }^{1}$, Batnaran Dagdan ${ }^{1,2}$, Solongo Battulga ${ }^{1}$, Lundiamaa Enkhbat ${ }^{1}$, \\ Nyamjav Byambasuren ${ }^{1}$, Munkhzol Malchinkhuu ${ }^{3}$ and Munkhtstetseg Janlav ${ }^{1, *}$ \\ 1 Department of Biochemistry, School of Biomedicine, Mongolian National University of Medical Sciences, \\ Ulaanbaatar 14210, Mongolia; ariunbold.md@gmail.com (A.C.-E.); Sengeragchaa.orgil@gmail.com (O.S.); \\ tsend-ayush@mnums.edu.mn (T.-A.A.); purevjal.s@gmail.com (P.S.); batnarandagdan@gmail.com (B.D.); \\ solongobattulga@gmail.com (S.B.); lundiae93@gmail.com (L.E.); bnyamjav0909@gmail.com (N.B.) \\ 2 Coronary Care Unit, Cardiovascular Center, The Shastin Central Hospital, Ulaanbaatar 16081, Mongolia \\ 3 Department of Pathophysiology, School of Biomedicine, Mongolian National University of Medical Sciences, \\ Ulaanbaatar 14210, Mongolia; munkhzol@mnums.edu.mn \\ * Correspondence: munkhtsetseg.j@mnums.edu.mn; Tel.: +976-9909-2287
}

Received: 27 July 2020; Accepted: 31 August 2020; Published: 2 September 2020

check for updates

\begin{abstract}
Metabolic syndrome (MetS) is complex and determined by the interaction between genetic and environmental factors and their influence on obesity, insulin resistance, and related traits associated with diabetes and cardiovascular disease risk. Some dynamic markers, including adiponectin $(A D I P O Q)$, brain-derived neurotrophic factor (BDNF), and lipoprotein lipase (LPL), are implicated in MetS; however, the influence of their genetic variants on MetS susceptibility varies in racial and ethnic groups. We investigated the association of single nucleotide polymorphism (SNP)-SNP interactions among nine SNPs in six genes with MetS's genetic predisposition in Mongolian subjects. A total of 160 patients with MetS for the case group and 144 healthy individuals for the control group were selected to participate in this study. Regression analysis of individual SNPs showed that the ADIPOQ $+45 \mathrm{GG}$ (odds ratio $(\mathrm{OR})=2.09, p=0.011)$ and $\mathrm{P}^{+} \mathrm{P}^{+}$of $L P L \mathrm{PvuII}(\mathrm{OR}$ $=2.10, p=0.038$ ) carriers had an increased risk of MetS. Conversely, G allele of LPL S447X (OR $=0.45, p=0.036)$ and PGC-1 $\alpha$ 482Ser $(\mathrm{OR}=0.26, p=0.001)$ allele were estimated as protective factors, respectively. Moreover, a haplotype containing the $\mathrm{G}-\mathrm{P}^{+}-\mathrm{G}$ combination was related to MetS. Significant loci were also related to body mass index (BMI), systolic blood pressure (SBP), serum high-density lipoprotein cholesterol (HDL-C), triglyceride (TG), and fasting blood glucose (FBG), adipokines, and insulin as well as insulin resistance $(p<0.05)$. Our results confirm that ADIPOQ + $45 \mathrm{~T}>\mathrm{G}, L P L$ PvII, and PGC-1 $\alpha$ Gly482Ser loci are associated with MetS in Mongolian subjects.
\end{abstract}

Keywords: metabolic syndrome; obesity; adipokines; insulin resistance

\section{Introduction}

Metabolic syndrome MetS is a substantial global public health problem and concern because of its high prevalence (20-25\% of the world's adult population) and linked to more severe pathologies [1]. Those with MetS are at a three-fold increased risk of developing cardiovascular diseases (CVDs) and five-fold increased risk of type 2 diabetes mellitus (T2DM) [2]. Since the precise definition of MetS differs in specific details issued by World Health Organization (WHO) in 1998 [3], The American Heart Association (AHA) in 2001 [4], the National Cholesterol Educational Program Expert Panel on Detection, Evaluation, and Treatment of High Blood Cholesterol in Adults (ATP III definition) in 
2001 [5], the National Heart, Lung and Blood Institute (NHLBI) in 2005 [6], and International Diabetes Federation (IDF) in 2006 [7], it is generally agreed that MetS is the compounding of several risk factors including abdominal obesity, insulin resistance, hyperglycemia, hyperlipidemia, and hypertension [1]. According to the IDF criteria, by 2015, the prevalence of MetS was estimated to occur in $32.7 \%$ in the general Mongolian population: socio-environmental factors including moderate-to-high alcohol consumption in men and widowed status in women are significantly associated with MetS [8].

The first-line intervention for MetS is to mitigate the modifiable, underlying risk factors (obesity, physical inactivity, and atherogenic diet) through lifestyle changes [9]. Effective lifestyle modification decreases the risk of developing type 2 diabetes among high-risk individuals by approximately $30-70 \%$ [10]. Then, if absolute risk is high enough, consideration can be given to incorporating drug therapy according to the existing guideline [11]. These reveal that the MetS, contrary to advanced type 2 diabetes and cardiovascular diseases, likely is a reversible condition if addressed early on, and long-term engagement in lifestyle changes may result in its resolution.

On the other hand, an irreversible risk factor is genetic background. Genetic influences play an essential role in the development of MetS in multiple ways, however, the mechanisms involved have not yet been fully understood. MetS's fundamental components (obesity [12], dyslipidemia [13], hyperglycemia [14], and high blood pressure (BP) [15]) have a genetic basis, for which candidate genes have been studied. A survey that comprised 44 single nucleotide polymorphism (SNP)s of 31 candidate genes related to the lipid metabolism among Japanese people with MetS found that $-3 \mathrm{~A}<\mathrm{G}$ and 553G < T (Gly185Cys) polymorphisms of Apolipoprotein A5 (APOA5), the 2052T < C (Val653Val) and 1866C < T (Asn591Asn) polymorphisms of Low-density lipoprotein receptor ( $L D L R)$, the 13989ARG (Ile118Val) polymorphism of CYP3A4 (Cytochrome P450 3A4) and the 1014T < A polymorphism of $\mathrm{C} 1 \mathrm{q}$ and tumor necrosis factor-related protein 5 (C1QTNF5) were significantly associated with the prevalence of MetS [16]. Some researchers suggested that population-specific SNP, related to body mass index (BMI), waist circumference (WC), or energy metabolism, are probably inherited among Mongolians as a positive selection [17]. Therefore, to determine the genetic risk among Mongolians, it is necessary to study the common gene polymorphisms associated with MetS.

To our knowledge, these gene polymorphisms have not been determined in the Mongolian subjects, and our study is the first attempt to investigate their relationships between gene polymorphisms and MetS in Mongolian subjects. The current study was intended to determine the genetic effect of some candidate gene polymorphisms on features of MetS in Mongolian subjects.

\section{Materials and Methods}

\subsection{Study Subjects}

All participants signed written informed consent. The protocol was according to the Helsinki Declaration, and ethical approval was obtained from the Ethics Committee at Mongolian National University of Medical Science (MNUMS; protocol \#201702/13-12/1A). A total of $160 \mathrm{MetS}$ patients (86 males, 74 females; aged: 18-60) were selected from the Ulaanbaatar population for study. Inclusion criteria were based on a modified or harmonizing criterion as proposed in 2009, by the IDF and the AHA-NHLBI [18] that incorporates ethnicity by providing different criteria for MetS in different ethnic groups; Asian identifies for subjects with at least three of the criteria listed: abdominal obesity with WC $\geq 90 \mathrm{~cm}$ for men and $\geq 80 \mathrm{~cm}$ for women, systolic blood pressure (SBP) $\geq 130 \mathrm{mmHg}$, diastolic blood pressure (DBP) $\geq 85 \mathrm{mmHg}$, serum triglyceride (TG) level $\geq 150 \mathrm{mg} / \mathrm{dL}$, serum high-density lipoprotein cholesterol (HDL-C) $<40 / 50 \mathrm{mg} / \mathrm{dL}$ for men/women, fasting blood glucose (FBG) $\geq 100 \mathrm{mg} / \mathrm{dL}$. The presence of subjects with independent diseases, including coronary heart disease, diabetes mellitus, or chronic diseases (hypertension, hyperlipidemia, and dyslipidemia), were excluded from the case group. The control group consisted of 144 individuals (71 males, 73 females; aged: 18-60) with no history of obesity, hyperlipidemia, dyslipidemia, hypertension, or diabetes mellitus and confirmed by a health examination. 


\subsection{Biochemical Parameters}

All blood samples were obtained from subjects after overnight fasting. Biochemical parameters were analyzed using commercial kits (AGAPPE DIAGNOSTICS SWITZERLAND GmbH, Knonauerstrasse 54-6330, Cham, Switzerland) for total cholesterol (TC), TG, HDL-C, and FBG. Low-density lipoprotein cholesterol (LDL-C) was calculated by the Friedewald formula. Homeostatic Model Assessment of Insulin Resistance (HOMA-IR) levels were measured by standard calculating methods: HOMA-IR $=$ glucose $(\mathrm{mg} / \mathrm{dL}) \times$ insulin $(\mu \mathrm{U} / \mathrm{mL}) / 405$. Serum adiponectin, leptin, and insulin levels were measured using a commercial direct enzyme-linked immunosorbent assay (ELISA) Human Adiponectin, Leptin, and Insulin kits, respectively, according to the procedure provided by the manufacturer (Linco Research, Inc., St. Louis, MO, USA).

\subsection{Genotyping of SNPs}

A total of nine SNPs: rs266729, rs2241766 of Adiponectin (ADIPOQ), rs6265 of Brain-derived neurotrophic factor $(B D N F)$, rs688, rs5925 of Low-density lipoprotein receptor (LDLR), rs1805094 of Leptin receptor (LEPR), rs285 and rs328 of Lipoprotein lipase (LPL), rs8192678 of Peroxisome proliferator-activated receptor gamma coactivator 1-alpha $(P G C-1 \alpha)$ were selected as our study targets. Genomic DNA was isolated by a commercial kit, the "G-spin ${ }^{\mathrm{TM}}$ Total DNA Extraction Kit" (iNtRON Biotechnology, Seongnam, South Korea). According to previously published protocols [19-26], all SNPs were genotyped by a polymerase chain reaction and restriction fragment length polymorphism (PCR-RFLP) using Maxine PCR PreMix Kit (i-Star Taq; iNtRON Biotechnology, Seongnam, South Korea). The list of primers used in this study was summarized in Table 1.

Table 1. Primers and restriction enzymes.

\begin{tabular}{|c|c|c|c|}
\hline Genes & SNPs & Primers & $\begin{array}{l}\text { Restriction } \\
\text { Enzymes }\end{array}$ \\
\hline \multirow[t]{2}{*}{$A D I P O Q$} & $-11377 \mathrm{C}>\mathrm{G}(\mathrm{rs} 266729)$ & $\begin{array}{c}\text { F: 5'-ACTTGCCCTGCCTCTGTCTG-3' R: } \\
\text { 5'-CCTGGAGAACTGGAAGCTG-3' }\end{array}$ & HhaI \\
\hline & $+45 \mathrm{~T}>\mathrm{G}(\mathrm{rs} 2241766)$ & $\begin{array}{l}\text { F: 5'-GAAGTAGACTCTGCTGAGATGG-3' } \\
\text { R: 5'-TATCAGTGTAGGAGGTCTGTGATG-3' }\end{array}$ & SmaI \\
\hline$B D N F$ & Val66Met (rs6265) & $\begin{array}{c}\text { F: 5'-ATCCGAGGACAAGGTGGC-3' } \\
\text { R: } \text { 5'-CCTCATGGACATGTTTGCAG-3' }^{\prime} \text {-CTTCA }\end{array}$ & Eco72I \\
\hline \multirow[t]{2}{*}{$L D L R$} & $\begin{array}{l}\text { C1773T } \\
\text { (rs688) }\end{array}$ & $\begin{array}{l}\text { F: 5'-TCTCCTTATCCACTTGTGTGT-3' } \\
\text { R: 5'-CTTCGATCTCGTACGTAAGC-3' }\end{array}$ & HincII \\
\hline & $\begin{array}{c}\text { AvalI } \\
\text { (rs5925) }\end{array}$ & $\begin{array}{l}\text { F: 5'-GTCATCTTCCTTGCTGCCTGTTTAG-3' } \\
\text { R: 5'-GTTTCCACAAGGAGGTTTCAAGGTT-3' }\end{array}$ & AvalI \\
\hline$L E P R$ & K656N (rs1805094) & $\begin{array}{l}\text { F: 5'-ACTAGATGGACTGGGATATTGGAGTAAT-3' } \\
\text { R: 5'-CTTCCAAAGTAAAGTGACATTTTTCGC -3' }\end{array}$ & BstUI \\
\hline$L P L$ & $\begin{array}{l}\text { PvuII } \\
(\mathrm{rs} 285) \\
\text { S447X } \\
(\mathrm{rs} 328)\end{array}$ & $\begin{array}{c}\text { F: 5'-ATCAGGCAATGCGTATGAGGTAA-3' } \\
\text { R: 5'-GAGACACAGATCTCTTAAGAC-3' } \\
\text { F: 5'-TACACTAGCAATGTCTAGGTGA-3' } \\
\text { R: 5'-TCAGCTTTAGCCCAGAATGC-3' }\end{array}$ & MnlI \\
\hline$P G C-1 \alpha$ & Gly482Ser (rs8192678) & $\begin{array}{l}\text { F: 5'-GAAGTCCTCAGTCCTCAC-3' R: } \\
\text { 5'-GGGGTCTTTGAGAAAATAAGG-3' }\end{array}$ & MspI \\
\hline
\end{tabular}

SNP, single nucleotide polymorphism; rs, reference SNP cluster id; ADIPOQ, Adiponectin; BDNF, Brain-derived neurotrophic factor; $L D L R$, Low-density lipoprotein receptor; $L E P R$, Leptin receptor; $L P L$, Lipoprotein lipase; $P G C-1 \alpha$, Peroxisome proliferator-activated receptor gamma coactivator 1-alpha.

\subsection{Statistical Analysis}

The quantitative data were represented as mean \pm standard deviation (SD) or median and interquartile range (IQR). Statistical significance was evaluated by a $t$-test to compare differences between the two groups after skewed distributed values were normalized by natural logarithmic transformation. Qualitative data were shown as a percentage, further analyzed by Chi-square $\left(\chi^{2}\right)$ test. The genotype distribution was compared between case and control groups with the $\chi^{2}$ 
test $(3 \times 2)$. The allele frequency was determined using direct gene counting analysis and $\chi^{2}$ test $(2 \times 2)$. For assessing the effect of the SNP genotype on the development of MetS, a multiple logistic regression analysis was performed with a $95 \%$ confidence interval (CI). In terms of the association of appreciable polymorphisms with clinical features, each polymorphism's genotype was transformed into a genetic model that constituted of two groups: a dominant group of wild-type homozygotes versus heterozygotes and homozygotes and the recessive group consisting of wild-type homozygotes and heterozygotes versus homozygotes. General data analyses of the case-control study were conducted using SPSS 21.0 (IBM corporation, Chicago, IL, USA). Haplotype and pairwise linkage disequilibrium (LD) analysis were carried out by SNPStats online software (Institut Català d'Oncologia, Barcelona, Spain; https://www.snpstats.net/start.htm).

\section{Results}

\subsection{Clinical Data and Biochemical Parameters}

The baseline profile of MetS patients and controls is summarized in Table 2. The mean age of patients with MetS was $41.7 \pm 11.3$ and the control group was $41.2 \pm 10.2$. The proportion of BMI $\geq$ $25 \mathrm{~kg} / \mathrm{m}^{2}$ among MetS and the control group was $64.1 \%$ and $35.9 \%$. Of the individuals with WC $\geq 80$ $\mathrm{cm}, 60.3 \%$ had MetS and $39.7 \%$ were in the control group. SBP and DBP levels were higher in patients with MetS than the control group $(p<0.001)$. Serum concentrations of FBG and TG were higher in the MetS group than the control group $(p=0.012, p<0.001)$. In contrast, HDL-C was lower $(p=0.048)$ in the MetS group than the control group. Our data illustrated that insulin, HOMA-IR and leptin levels were also higher in the MetS group $(p<0.001)$.

Table 2. Main characteristics of the MetS and control group.

\begin{tabular}{cccc}
\hline Parameters & MetS & Control & $p$-Value \\
\hline Age, yr & $41.7 \pm 11.3$ & $41.2 \pm 10.2$ & 0.924 \\
Gender $(\mathrm{M} / \mathrm{F})$ & $160(86 / 74)$ & $144(71 / 73)$ & 0.527 \\
BMI, kg/m ${ }^{2}$ & $31.27 \pm 4.23$ & $26.64 \pm 3.75$ & $<0.001$ \\
WC, cm & $100.97 \pm 1.10$ & $89.01 \pm 12.75$ & $<0.001$ \\
SBP, $\mathrm{mmHg}$ & $128.75 \pm 13.97$ & $114.02 \pm 14.44$ & $<0.001$ \\
DBP, mmHg & $88.42 \pm 9.92$ & $77.95 \pm 9.52$ & $<0.001$ \\
FBG, mg/dL & $92.07 \pm 66.57$ & $71.69 \pm 12.69$ & 0.012 \\
TC, $\mathrm{mg} / \mathrm{dL}$ & $157.72 \pm 36.42$ & $148.47 \pm 36.73$ & 0.123 \\
TG, mg/dL 1 & $124.45(83.74-179.37)$ & $66.37(49.52-96.35)$ & $<0.001$ \\
HDL-C, mg/dL & $32.04 \pm 11.49$ & $36.44 \pm 15.63$ & 0.048 \\
LDL-C, mg/dL & $96.85 \pm 40.38$ & $95.75 \pm 39.53$ & 0.867 \\
Adiponectin, ng/mL ${ }^{1}$ & $6.46(0.06-19.51)$ & $6.18(0.09-49.66)$ & 0.082 \\
Leptin, $\mathrm{ng} / \mathrm{mL}^{1}$ & $11.10(2.30-56.30)$ & $4.5(0.01-34.87)$ & $<0.001$ \\
Insulin, mIU/mL ${ }^{1}$ & $12.06(0.29-113.53)$ & $8.53(0.29-170.29)$ & $<0.001$ \\
HOMA-IR ${ }^{1}$ & $2.28(0.05-38.99)$ & $1.43(0.05-36.32)$ & $<0.001$ \\
\hline
\end{tabular}

MetS, metabolic syndrome; BMI, body mass index; M, male; F, female; WC, waist circumference; SBP, systolic blood pressure; DBP, diastolic blood pressure; FBG, fasting blood glucose; TG, triglyceride; TC, total cholesterol; HDL-C, high-density lipoprotein cholesterol; LDL-C, low-density lipoprotein cholesterol; HOMA-IR, homeostatic model assessment-insulin resistance. Values for continuous variables are expressed as the mean \pm standard deviation (SD) and the median and interquartile range (IQR). ${ }^{1}$ After log-transformed, the $t$-test was utilized for comparison. 


\subsection{Allele Frequency of SNPS}

Genotype frequencies of nine SNPs in the MetS group and control group were calculated (Table 3). The $\chi^{2}$ test revealed that four of the identified SNPs were significantly related to the prevalence of MetS.

Table 3. Genotype frequencies of SNPs.

\begin{tabular}{|c|c|c|c|c|c|}
\hline Genes & SNPs & Genotype & MetS $n(\%)$ & $\begin{array}{c}\text { Control Group } \\
n(\%)\end{array}$ & $p$-Value \\
\hline \multirow[t]{6}{*}{$A D I P O Q$} & \multirow{3}{*}{$\begin{array}{c}-11377 C>G \\
(r s 266729)\end{array}$} & $\mathrm{CC}$ & $82(51.3)$ & $71(49.3)$ & \multirow{3}{*}{0.775} \\
\hline & & CG & 70 (43.6) & 63 (43.7) & \\
\hline & & GG & $8(5.1)$ & $10(7.0)$ & \\
\hline & \multirow{3}{*}{$\begin{array}{l}+45 \mathrm{~T}>\mathrm{G} \\
(\mathrm{rs} 2241766)\end{array}$} & $\mathrm{TT}$ & $72(45.0)$ & $85(59.0)$ & \multirow{3}{*}{0.002} \\
\hline & & TG & $80(50.0)$ & 44 (30.5) & \\
\hline & & GG & $8(5.0)$ & 15 (10.4) & \\
\hline \multirow[t]{3}{*}{$B D N F$} & \multirow{3}{*}{$\begin{array}{l}\text { Val66Met } \\
\text { (rs6265) }\end{array}$} & Met/Met & $33(21.2)$ & $54(37.5)$ & \multirow{3}{*}{0.157} \\
\hline & & Val/Met & $119(73.8)$ & $84(58.3)$ & \\
\hline & & $\mathrm{Val} / \mathrm{Val}$ & $8(5)$ & $6(4.2)$ & \\
\hline \multirow[t]{6}{*}{$L D L R$} & $\mathrm{C} 1773 \mathrm{~T}$ & $\mathrm{CC}$ & $2(1.2)$ & $4(2.8)$ & \multirow{3}{*}{0.578} \\
\hline & \multirow{2}{*}{ (rs688) } & CT & 97 (60.9) & 66 (45.7) & \\
\hline & & TT & $61(37.8)$ & 74 (51.4) & \\
\hline & \multirow{3}{*}{$\begin{array}{c}\text { AvalI } \\
\text { (rs5925) }\end{array}$} & $\mathrm{A}^{-} \mathrm{A}^{-}$ & $90(56.2)$ & $71(49.3)$ & \multirow{3}{*}{0.976} \\
\hline & & $\mathrm{A}^{-} \mathrm{A}^{+}$ & $70(43.8)$ & $65(45.1)$ & \\
\hline & & $\mathrm{A}^{+} \mathrm{A}^{+}$ & - & $8(5.6)$ & \\
\hline \multirow[t]{3}{*}{$L E P R$} & \multirow{3}{*}{$\begin{array}{c}\mathrm{K} 656 \mathrm{~N} \\
\text { (rs1805094) }\end{array}$} & GG & $128(76.3)$ & $110(76.4)$ & \multirow{3}{*}{0.865} \\
\hline & & GC & $26(16.3)$ & $26(18.1)$ & \\
\hline & & $\mathrm{CC}$ & $6(7.5)$ & $8(5.6)$ & \\
\hline \multirow[t]{6}{*}{$L P L$} & PvuII & $\mathrm{P}^{-} \mathrm{P}^{-}$ & $51(31.8)$ & $40(27.8)$ & \multirow{3}{*}{0.028} \\
\hline & \multirow[t]{2}{*}{ (rs285) } & $\mathrm{P}^{-} \mathrm{P}^{+}$ & $50(31.3)$ & $66(45.8)$ & \\
\hline & & $\mathrm{P}^{+} \mathrm{P}^{+}$ & $59(36.9)$ & $38(26.4)$ & \\
\hline & \multirow{3}{*}{$\begin{array}{l}\text { S447X } \\
\text { (rs328) }\end{array}$} & $\mathrm{CC}$ & $143(89.3)$ & $106(73.6)$ & \multirow{3}{*}{$<0.001$} \\
\hline & & CG & $16(10.0)$ & 33 (22.9) & \\
\hline & & GG & $1(6.2)$ & $5(3.4)$ & \\
\hline \multirow[t]{3}{*}{$P G C-1 \alpha$} & Gly482Ser & GG & $83(51.8)$ & $48(33.4)$ & \multirow{3}{*}{0.004} \\
\hline & (rs8192678) & GS & $55(34.3)$ & 69 (47.9) & \\
\hline & & SS & 22 (13.7) & 27 (18.7) & \\
\hline
\end{tabular}

SNPs, single nucleotide polymorphisms; MetS, metabolic syndrome. Genotype distributions of each SNP were compared between case and control groups using the $\chi^{2}$ test $(3 \times 2)$.

Results of the multiple logistic regression model, used to determine the significance of alleles of the two SNPs as a probable independent risk factor for MetS, demonstrated that heterozygous (TG + GG) of $A D I P O Q+45 \mathrm{~T}>\mathrm{G}$ (odds ratio $(\mathrm{OR})=1.98 ; 95 \% \mathrm{CI}, 1.14-3.44 ; p=0.015)$, and homozygous $\left(\mathrm{P}^{+} \mathrm{P}^{+}\right)$of LPL PvuII (OR $=2.10 ; 95 \% \mathrm{CI}, 1.04-4.26 ; p=0.038)$ carriers had an increased risk for development of MetS compared with those wild-type or the most frequent genotypes (Table 4). On the other hand, heterozygotes (CG + GG) of LPL S447X (OR = 0.45; 95\%CI, 0.21-0.95; $p=0.036)$ and homozygotes (SS) of PGC- $1 \alpha$ Gly482Ser (OR $=0.26 ; 95 \% \mathrm{CI}, 0.12-0.58 ; p=0.001$ ) were independently estimated as a protective factor for MetS. 
Table 4. Genotype frequencies of polymorphisms associated with MetS.

\begin{tabular}{|c|c|c|c|c|c|}
\hline Gene/SNP & Genotype & MetS $n(\%)$ & Control Group $n(\%)$ & OR * $(95 \% C I)$ & $p$-Value \\
\hline \multirow{5}{*}{$\begin{array}{l}A D I P O Q+45 \mathrm{~T} \\
>\mathrm{G}(\mathrm{rs} 2241766)\end{array}$} & TT & $72(45.0)$ & $85(59.0)$ & 1.00 & \\
\hline & TG & $80(50.0)$ & $44(30.5)$ & $1.39(0.44-3.18)$ & 0.570 \\
\hline & GG & $8(5.0)$ & $15(10.4)$ & $2.09(1.18-3.72)$ & 0.011 \\
\hline & $\mathrm{TT} / \mathrm{TG}+\mathrm{GG}$ & $88(55.0)$ & $59(41.0)$ & $1.98(1.14-3.44)$ & 0.015 \\
\hline & $\mathrm{TT}+\mathrm{TG} / \mathrm{GG}$ & $8(5.0)$ & $15(10.4)$ & $0.53(0.18-1.10)$ & 0.081 \\
\hline \multirow{5}{*}{$\begin{array}{l}\text { LPL PvuII } \\
\quad(\mathrm{rs} 285)\end{array}$} & $\mathrm{P}^{-} \mathrm{P}^{-}$ & $51(31.8)$ & $40(27.8)$ & 1.00 & \\
\hline & $\mathrm{P}^{-} \mathrm{P}^{+}$ & $50(31.3)$ & $66(45.8)$ & $0.85(0.43-1.69)$ & 0.661 \\
\hline & $\mathrm{P}^{+} \mathrm{P}^{+}$ & $59(36.9)$ & $38(26.4)$ & $2.10(1.04-4.26)$ & 0.038 \\
\hline & $\begin{array}{c}\mathrm{P}^{-} \mathrm{P}^{-} / \mathrm{P}^{-} \mathrm{P}^{+}+ \\
\mathrm{P}^{+} \mathrm{P}^{+}\end{array}$ & $109(68.1)$ & $104(72.2)$ & $1.31(0.72-2.39)$ & 0.370 \\
\hline & $\begin{array}{c}\mathrm{P}^{-} \mathrm{P}^{-}+ \\
\mathrm{P}^{-} \mathrm{P}^{+} / \mathrm{P}^{+} \mathrm{P}^{+}\end{array}$ & $59(36.9)$ & $38(26.4)$ & $2.29(1.26-4.18)$ & 0.006 \\
\hline \multirow{5}{*}{$\begin{array}{l}\text { LPL S447X } \\
\quad(\mathrm{rs} 328)\end{array}$} & $\mathrm{CC}$ & $143(89.3)$ & $106(73.6)$ & 1.00 & \\
\hline & CG & $16(10.0)$ & $33(22.9)$ & $0.17(0.02-1.54)$ & 0.117 \\
\hline & GG & $1(6.2)$ & $5(3.4)$ & $0.52(0.23-1.15)$ & 0.106 \\
\hline & $\mathrm{CC} / \mathrm{CG}+\mathrm{GG}$ & $17(10.6)$ & $38(26.4)$ & $0.45(0.21-0.95)$ & 0.036 \\
\hline & $\mathrm{CC}+\mathrm{CG} / \mathrm{GG}$ & $1(6.2)$ & $5(3.4)$ & $0.19(0.02-1.70)$ & 0.139 \\
\hline \multirow{5}{*}{$\begin{array}{c}\text { PGC1 } \\
\text { Gly482Ser } \\
\text { (rs8192678) }\end{array}$} & GG & $83(51.8)$ & $48(33.4)$ & 1.00 & \\
\hline & GS & $55(34.3)$ & $69(47.9)$ & $0.41(0.22-0.77)$ & 0.006 \\
\hline & SS & $22(13.7)$ & $27(18.7)$ & $0.26(0.12-0.58)$ & 0.001 \\
\hline & $\mathrm{GG} / \mathrm{GS}+\mathrm{SS}$ & $77(48.1)$ & $96(66.7)$ & $0.36(0.20-0.63)$ & $<0.001$ \\
\hline & $\mathrm{GG}+\mathrm{GS} / \mathrm{SS}$ & $22(13.7)$ & $27(18.7)$ & $0.42(0.20-0.83)$ & 0.013 \\
\hline
\end{tabular}

MetS, metabolic syndrome; SNP, single nucleotide polymorphism; OR, odds ratio; $\mathrm{CI}$, confidence interval. *Adjusted with age, gender, BMI and WC.

\subsection{SNP-SNP Interaction and MetS}

We analyzed the pairwise linkage disequilibrium (LD) pattern for significant polymorphisms: $A D I P O Q+45 \mathrm{~T}>\mathrm{G}$ (rs2241766), LPL PvuII (rs285), and PGC-1 $\alpha$ Gly482Ser (rs8192678) (Figure 1). No SNP combination was in strong $L D\left(\mathrm{D}^{\prime}>0.7, \mathrm{r}^{2}>0.25\right)$. Furthermore, we carried out haplotype analysis using multiple logistic regression models and listed all combinations accounted for more than $5 \%$ of the frequency (Table 5). Out of possible variants, we found (G-P $-\mathrm{G}$ ) haplotype significantly associated with the development of MetS (OR $=3.28 ; 95 \% \mathrm{CI}, 1.32-8.16 ; p=0.011)$.

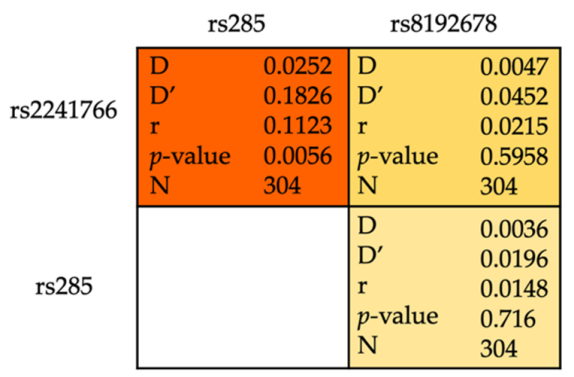

Figure 1. Paired single nucleotide polymorphism (SNP) linkage disequilibrium analysis.

Table 5. Association of Haplotype with MetS risk.

\begin{tabular}{|c|c|c|c|c|c|c|c|}
\hline & \multirow{2}{*}{ rs2241766 } & \multirow{2}{*}{ rs285 } & \multirow{2}{*}{ rs8192678 } & \multicolumn{2}{|c|}{ Frequency } & \multirow{2}{*}{$\mathrm{OR} *(95 \% \mathrm{CI})$} & \multirow{2}{*}{$p$-value } \\
\hline & & & & MetS & Control & & \\
\hline 1 & $\mathrm{~T}$ & $\mathrm{P}^{-}$ & G & 0.264 & 0.249 & 1.00 & - \\
\hline 2 & $\mathrm{~T}$ & $\mathrm{P}^{+}$ & G & 0.204 & 0.168 & $0.83(0.40-1.73)$ & 0.630 \\
\hline 3 & $\mathrm{~T}$ & $\mathrm{P}^{+}$ & $\mathrm{S}$ & 0.124 & 0.182 & $1.27(0.65-2.51)$ & 0.49 \\
\hline 4 & G & $\mathrm{P}^{+}$ & G & 0.152 & 0.100 & $3.28(1.32-8.16)$ & 0.011 \\
\hline 5 & G & $\mathrm{P}^{-}$ & G & 0.066 & 0.05 & $1.39(0.30-6.48)$ & 0.683 \\
\hline 6 & G & $\mathrm{P}^{-}$ & $\mathrm{S}$ & 0.044 & 0.063 & $1.58(0.49-5.07)$ & 0.440 \\
\hline 7 & G & $\mathrm{P}^{+}$ & S & 0.037 & 0.042 & $1.26(0.24-6.65)$ & 0.788 \\
\hline
\end{tabular}

MetS, metabolic syndrome; OR, odds ratio; CI, confidence interval. * Adjusted with age, gender, BMI and WC. 


\subsection{Association of SNPs with Clinical Parameters}

Clinical characteristics of all enrolled people were compared according to the dominant and recessive genotype of the SNPs relevant to MetS (Table 6). Our results suggest that the HDL-C and adiponectin levels were reduced more in $A D I P O Q+45 \mathrm{~T}>\mathrm{G}$ carriers (TG + GG) than those non-carriers (TT) in the dominant model ( $p=0.032, p=0.027$, respectively). In the recessive, we found that BMI and blood pressure levels were higher in the homozygous carrier group (GG; $p=0.012, p=0.001$ ) of the SNP.

For LPL PvuII, $\left(\mathrm{P}^{+} \mathrm{P}^{+}\right)$genotype carriers had elevated TG and FBG levels compared with combined genotypes $\left(\mathrm{P}^{+} \mathrm{P}^{-}+\mathrm{P}^{-} \mathrm{P}^{-} ; p=0.009, p<0.001\right)$. Meanwhile, $\left(\mathrm{P}^{+}\right)$allele $\left(\mathrm{P}^{+} \mathrm{P}^{+}+\mathrm{P}^{+} \mathrm{P}^{-}\right)$in the recessive model showed elevated BMI and insulin levels than compared to non-carrier homozygotes $\left(\mathrm{P}^{-} \mathrm{P}^{-}\right.$; $p=0.027, p=0.027)$. Whereas $\left(\mathrm{P}^{+}\right)$allele carriers seemingly had more leptin secretion in both models $(p=0.001, p<0.001)$.

With regard to the PGC-1 $\alpha$ Gly482Ser, the recessive (SS) genotype carriers had elevated levels of SBP than $(G)$ allele carriers $(p=0.004, p=0.012)$ but our results disclosed higher levels of HOMA-IR in $(\mathrm{GG}+\mathrm{GS})$ genotype carriers which are opposite of levels found in (SS) genotype carriers $(p=0.038)$. Moreover, (GG) homozygotes were significantly associated with low levels of HDL-C $(p=0.011)$.

Table 6. Clinical features of subjects according to the genotype of polymorphisms.

\begin{tabular}{|c|c|c|c|c|c|c|c|}
\hline \multirow{2}{*}{ SNP } & \multirow{2}{*}{ Parameters } & \multicolumn{2}{|c|}{ Dominant Model a $^{\text {a }}$} & \multicolumn{4}{|c|}{ Recessive Model $^{\text {b }}$} \\
\hline & & AA & $\mathbf{A b}+\mathbf{b b}$ & $p$-Value & $\mathbf{A A}+\mathbf{A b}$ & $\mathbf{b b}$ & $p$-value \\
\hline \multirow{12}{*}{ 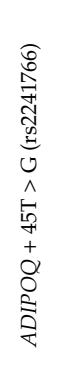 } & BMI, $\mathrm{kg} / \mathrm{m}^{2}$ & $28.92 \pm 4.41$ & $29.32 \pm 4.83$ & 0.450 & $26.69 \pm 5.29$ & $29.29 \pm 4.52$ & 0.012 \\
\hline & $\mathrm{WC}, \mathrm{cm}$ & $90.57 \pm 9.13$ & $95.80 \pm 13.48$ & 0.082 & $94.82 \pm 13.51$ & $96.08 \pm 13.03$ & 0.412 \\
\hline & $\mathrm{SBP}, \mathrm{mmHg}$ & $121.59 \pm 17.18$ & $122.58 \pm 14.55$ & 0.597 & $111.42 \pm 8.38$ & $122.91 \pm 16.06$ & 0.001 \\
\hline & $\mathrm{TC}, \mathrm{mg} / \mathrm{dL}$ & $149.76 \pm 37.80$ & $156.62 \pm 35.40$ & 0.105 & $152.50 \pm 32.58$ & $153.37 \pm 37.05$ & 0.913 \\
\hline & $\mathrm{TG}, \mathrm{mg} / \mathrm{dL}^{1}$ & $82.74(55.93-149.90)$ & $94.35(66.34-139.40)$ & 0.629 & $90.09(66.68-169.40)$ & $93.40(56.64-143.40)$ & 0.845 \\
\hline & HDL-C, mg/dL & $35.66 \pm 14.47$ & $32.40 \pm 12.62$ & 0.032 & $33.68 \pm 13.00$ & $38.78 \pm 14.85$ & 0.086 \\
\hline & LDL-C, mg/dL & $97.04 \pm 40.96$ & $95.44 \pm 38.52$ & 0.730 & $96.62 \pm 40.00$ & $92.03 \pm 36.98$ & 0.595 \\
\hline & FBG, $\mathrm{mg} / \mathrm{dL}$ & $77.68 \pm 39.12$ & $87.47 \pm 59.01$ & 0.088 & $81.87 \pm 50.15$ & $89.06 \pm 47.26$ & 0.508 \\
\hline & Adiponectin, $\mathrm{ng} / \mathrm{mL}^{1}$ & $6.41(4.05-10.38)$ & $6.33(2.82-10.18)$ & 0.027 & $6.51(4.02-10.31)$ & $5.28(3.42-12.91)$ & 0.660 \\
\hline & $\begin{array}{l}\text { Leptin, } \\
\mathrm{ng} / \mathrm{mL}^{1}\end{array}$ & $7.5(4.37-15.75)$ & $7.2(3.85-19.87)$ & 0.559 & $7.20(4.34-16.46)$ & $7.41(2.12-18.74)$ & 0.127 \\
\hline & $\begin{array}{l}\text { Insulin, } \\
\mathrm{mIU} / \mathrm{mL}^{1}\end{array}$ & $10.88(5.88-15.59)$ & $11.18(5.59-17.06)$ & 0.736 & $11.18(5.59-16.62)$ & $9.12(5.29-13.82)$ & 0.256 \\
\hline & HOMA-IR $^{1}$ & $1.87(0.97-3.10)$ & $1.84(1.04-3.57)$ & 0.828 & $1.87(1.03-3.22)$ & $1.81(0.98-3.04)$ & 0.468 \\
\hline \multirow{12}{*}{ 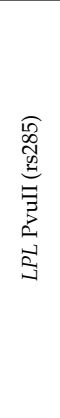 } & BMI, $\mathrm{kg} / \mathrm{m}^{2}$ & $29.42 \pm 4.89$ & $28.45 \pm 3.91$ & 0.088 & $30.00 \pm 4.83$ & $28.72 \pm 4.47$ & 0.027 \\
\hline & $\mathrm{WC}, \mathrm{cm}$ & $95.45 \pm 11.26$ & $95.42 \pm 14.16$ & 0.984 & $97.40 \pm 13.53$ & $94.55 \pm 13.09$ & 0.088 \\
\hline & $\mathrm{SBP}, \mathrm{mmHg}$ & $123.19 \pm 17.88$ & $121.56 \pm 14.90$ & 0.416 & $122.40 \pm 16.39$ & $121.36 \pm 14.79$ & 0.610 \\
\hline & $\mathrm{TC}, \mathrm{mg} / \mathrm{dL}$ & $157.36 \pm 34.00$ & $151.47 \pm 37.76$ & 0.197 & $152.22 \pm 36.40$ & $155.78 \pm 37.39$ & 0.438 \\
\hline & $\mathrm{TG}, \mathrm{mg} / \mathrm{dL}^{1}$ & $\begin{array}{c}130.75 \\
(86.74-187.50)\end{array}$ & $\begin{array}{c}117.30 \\
(82.74-174.80)\end{array}$ & 0.009 & $\begin{array}{c}113.60 \\
(78.31-185.40)\end{array}$ & $\begin{array}{c}150.80 \\
(97.98-176.32)\end{array}$ & 0.787 \\
\hline & HDL-C, mg/dL & $32.87 \pm 14.10$ & $34.65 \pm 13.47$ & 0.296 & $33.99 \pm 13.32$ & $34.27 \pm 14.51$ & 0.870 \\
\hline & LDL-C, mg/dL & $97.37 \pm 39.54$ & $95.75 \pm 39.91$ & 0.745 & $99.01 \pm 41.22$ & $95.02 \pm 39.08$ & 0.425 \\
\hline & $\mathrm{FBG}, \mathrm{mg} / \mathrm{dL}$ & $99.90 \pm 79.70$ & $74.34 \pm 22.85$ & $<0.001$ & $85.01 \pm 56.51$ & $76.44 \pm 28.89$ & 0.082 \\
\hline & Adiponectin, $\mathrm{ng} / \mathrm{mL}^{1}$ & $5.60(4.05-7.21)$ & $7.23(5.20-10.01)$ & 0.078 & $6.23(4.33-9.74)$ & $7.11(5.17-8.98)$ & 0.057 \\
\hline & $\begin{array}{l}\text { Leptin, } \\
\mathrm{ng} / \mathrm{mL}^{1}\end{array}$ & $14.65(7.50-27.80)$ & $9.60(6.40-22.60)$ & 0.001 & $15.95(7.50-25.10)$ & $7.80(4.60-9.70)$ & $<0.001$ \\
\hline & $\begin{array}{l}\text { Insulin, } \\
\mathrm{mIU} / \mathrm{mL}^{1}\end{array}$ & $13.97(7.94-18.53)$ & $11.76(5.59-21.76)$ & 0.113 & $12.06(7.35-21.76)$ & $11.47(6.03-19.78)$ & 0.021 \\
\hline & HOMA-IR $^{1}$ & $2.32(1.19-4.06)$ & $2.28(1.23-4.26)$ & 0.997 & $2.31(1.64-4.37)$ & $2.23(1.11-4.07)$ & 0.194 \\
\hline \multirow{12}{*}{ 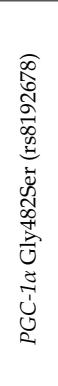 } & BMI, $\mathrm{kg} / \mathrm{m}^{2}$ & $29.13 \pm 4.71$ & $29.10 \pm 4.55$ & 0.958 & $29.00 \pm 4.87$ & $29.65 \pm 2.99$ & 0.368 \\
\hline & $\mathrm{WC}, \mathrm{cm}$ & $95.97 \pm 14.29$ & $95.01 \pm 12.46$ & 0.537 & $98.12 \pm 10.76$ & $94.89 \pm 13.68$ & 0.117 \\
\hline & $\mathrm{SBP}, \mathrm{mmHg}$ & $125.07 \pm 16.37$ & $119.75 \pm 15.18$ & 0.004 & $122.93 \pm 16.59$ & $118.00 \pm 11.33$ & 0.012 \\
\hline & $\mathrm{TC}, \mathrm{mg} / \mathrm{dL}$ & $155.64 \pm 37.50$ & $150.22 \pm 35.48$ & 0.204 & $154.37 \pm 35.98$ & $147.97 \pm 39.99$ & 0.261 \\
\hline & $\mathrm{TG}, \mathrm{mg} / \mathrm{dL}^{1}$ & $99.08(56.36-169.25)$ & $90.57(59.83-129.20)$ & 0.137 & $94.35(56.92-147.40)$ & $86.74(59.60-121.55)$ & 0.404 \\
\hline & HDL-C, mg/dL & $31.76 \pm 11.65$ & $35.82 \pm 14.82$ & 0.011 & $33.48 \pm 12.91$ & $37.04 \pm 16.83$ & 0.092 \\
\hline & LDL-C, mg/dL & $98.71 \pm 41.50$ & $93.05 \pm 37.20$ & 0.226 & $97.39 \pm 39.06$ & $90.73 \pm 42.86$ & 0.281 \\
\hline & $\mathrm{FBG}, \mathrm{mg} / \mathrm{dL}$ & $84.46 \pm 59.93$ & $80.89 \pm 40.96$ & 0.538 & $82.60 \pm 52.33$ & $81.45 \pm 35.46$ & 0.881 \\
\hline & Adiponectin, $\mathrm{ng} / \mathrm{mL}^{1}$ & $6.28(4.02-10.61)$ & $6.68(3.50-10.06)$ & 0.087 & $6.33(3.77-10.46)$ & $6.95(2.46-8.85)$ & 0.314 \\
\hline & $\begin{array}{l}\text { Leptin, } \\
\mathrm{ng} / \mathrm{mL}^{1}\end{array}$ & $7.20(4.40-16.46)$ & $7.01(3.90-17.30)$ & 0.210 & $7.00(3.91-15.67)$ & $8.51(4.71-20.24)$ & 0.519 \\
\hline & $\begin{array}{l}\text { Insulin, } \\
\mathrm{mIU} / \mathrm{mL}^{1}\end{array}$ & $11.18(6.39-14.48)$ & $10.29(5.29-17.06)$ & 0.830 & $10.29(5.59-14.41)$ & $12.35(7.86-21.03)$ & 0.067 \\
\hline & HOMA-IR $^{1}$ & $1.76(1.10-2.90)$ & $1.95(0.91-3.43)$ & 0.142 & $1.81(0.98-3.04)$ & $2.52(1.25-4.39)$ & 0.038 \\
\hline
\end{tabular}

Values for continuous variables are expressed as the mean \pm standard deviation (SD) and as the median and interquartile range (IQR). ${ }^{1}$ After log-transformed, the $t$-test was utilized for comparison. ${ }^{\text {a }}$ Dominant model TT/TG $+\mathrm{GG}$ for $A D I P O Q+45 \mathrm{~T}>\mathrm{G}, \mathrm{P}^{+} \mathrm{P}^{+} / \mathrm{P}^{-} \mathrm{P}^{+}+\mathrm{P}^{-} \mathrm{P}^{-}$for $L P L$ PvuII, GG/GS + SS for PGC-1 $\alpha$ Gly 482 Ser. ${ }^{\mathrm{b}}$ Recessive model $\mathrm{TT}+\mathrm{TG} / \mathrm{GG}$ for $A D I P O Q+45 \mathrm{~T}>\mathrm{G}, \mathrm{P}^{+} \mathrm{P}^{+}+\mathrm{P}^{-} \mathrm{P}^{+} / \mathrm{P}^{-} \mathrm{P}^{-}$for $L P L$ PvuII, GG + GS/SS for PGC-1 $\alpha$ Gly482Ser. 


\section{Discussion}

The estimation of environmental and genetic influences on each MetS component may vary in different populations and ethnicities. Some genome-wide prediction surveys focused on associations with the MetS or clinical criteria $[27,28]$. Whereas, other studies of population-specific SNPs that were selected for their potential contribution to carbohydrate and lipid metabolism found that some gene polymorphisms were strongly related to FBG and TG levels [29,30]. We have investigated the gene variants affecting dynamic markers: $B D N F, L E P R, V L D L R$, and $P G C-1 \alpha$, but included loci related to cellular lipid metabolism, $L P L$ and $A D I P O Q$ were also identified in Mongolian patients with MetS. Only four out of nine tested gene variants, the ADIPOQ + 45T > G, LPL PvuII and S447X, PGC-1 $\alpha$ Gly482Ser, showed a nominal difference in the initial genotype comparison.

\subsection{Adiponectin (ADIPOQ)}

The effects of genetic polymorphisms of $A D I P O Q$ on the risk of obesity, T2D, and hypertension incidence have been studied in several ethnic groups. Among the Chinese Han population, ADIPOQ $+45 \mathrm{~T}>\mathrm{G}$ has been determined to be related to MetS [31]. Similarly, we have now shown that the ADIPOQ $+45 \mathrm{~T}>\mathrm{G}$ is associated with MetS. Moreover, we found significant associations between the polymorphism of the $A D I P O Q$ and BMI, SBP, and HDL-C levels in the genetic models apart from the adiponectin level (Table 6). Our results are in agreement with other findings from the meta-analysis surveys that indicate $A D I P O Q+45 \mathrm{~T}>\mathrm{G}$ polymorphism is associated with hypertension and dyslipidemia phenotypes [31]. A silent mutation, from the changing substitution of $(\mathrm{T})$ with $(\mathrm{G})$ in the $+45 \mathrm{~T}>\mathrm{G}$ polymorphism that occurs in exon 2, may affect transcription rate, splicing, and mRNA transport regulation, probably explain this association. A haplotype analysis, based on a dense SNP map in a large sample of non-Hispanic whites and African Americans, clarified gene interaction involving haplotypes in the promoter and coding region, a 2-block linkage disequilibrium structure of the $A D I P O Q$ impacting the level of plasma adiponectin [32]. From the survey, blocks have at least one SNP related to serum adiponectin levels. The haplotypes in the first block were linked to increased adiponectin levels. In contrast, the haplotypes in the second one were related to decreased adiponectin levels. Hence, the $+45 \mathrm{~T}>\mathrm{G}$ polymorphism is significantly associated with reduced serum adiponectin levels and high blood pressure. However, the mechanism of whether the $+45 \mathrm{~T}>\mathrm{G}$ polymorphism influences the hypertension susceptibility through the low levels of plasma adiponectin warrants further study. Another study, inversely, did not detect such effects or provided contradictory results concerning the polymorphic sites that are involved with an alteration in blood lipids [33] and glucose [34]. Nevertheless, it should be noted that several recent genome-wide association studies (GWAS) have been carried out on MetS components: obesity, dyslipidemia, high BP and T2D individually in several populations, most of which were conducted in people of European descent. The interactions between genetic factors, such as SNPs in the Adiponectin itself, and environmental factors causing obesity, may play a crucial role in developing insulin resistance, type 2 diabetes, and the MetS.

\subsection{Lipoprotein Lipase (LPL)}

The PvuII and S447X polymorphisms are located on intron 1 and exon 9, respectively. The genetic association has been described for PvuII and S447X polymorphisms in the LPL gene with various pathological conditions, including dyslipidemia, hypertension, CVD, and T2D. The meta-analysis study revealed a significant protective association between Ser447Ter and PvuII and the stroke risk [35]. Our findings indicated that PvuII is a risk factor for MetS, while the S447X has an inverse effect. In terms of PvuII, we also found increased levels of BMI and FBG. A similar tendency was reported by Bozina et al. [36], where they indicated that the PvuII $\left(\mathrm{P}^{+} \mathrm{P}^{+}\right)$genotype carrier had a higher level of BMI and glucose. Besides, the combination of two mutant alleles in HindIII (rs320) and PvuII was related to the increased level of WC [37] a potential indicator of visceral fat accumulation, which is closely associated with obesity-related MetS. Interestingly, increased levels of TG, leptin and insulin 
were recorded in PvuII $\left(\mathrm{P}^{+}\right)$allele carriers in our study. This coincidence might depict a dysregulation of energy homeostasis in coordinated leptin and insulin resistance due to elevated TG levels. A recent report indicates that an inhibition of leptin receptor by the serum TG in the brain induces central resistance to leptin and insulin for their centrally mediated effect on body weight [38], which probably is consistent with our findings. Concerning the S447X polymorphism, the only case of (GG) genotype was observed in the MetS group in the current study, except for combination genotype frequency. Due to that, we did not analyze it with biochemical parameters.

\subsection{Peroxisome Proliferator-Activated Receptor Gamma Coactivator 1-Alpha (PPARGC1 $\alpha$ or PGC-1 $\alpha$ )}

Combining the initial and the replication analysis presented a 0.26-0.42-fold decrease in MetS risk associated with the (S) allele in our study. At variance, this result is the only negative study published thus far [39], in which no association was reported in Danish subjects with MetS. We found a significant association between PGC-1 $\alpha$ Gly482Ser polymorphism and insulin resistance or HOMA-IR and SBP as well as HDL-C in the genetic model analysis (Table 4). Interestingly, our study indicated that the (S) allele was estimated as a protector factor for MetS, while the (S) allele carriers are more insulin resistant. Our results are consistent with the previous study reporting an association with insulin resistance [40]. However, we should point out a possible explanation for this issue because patients with the MetS may already be the result of insulin resistance, making it more complicated to distinguish between subjects within the MetS group. PGC-1 $\alpha$ enhances the activity of other PPARs that bind to sequence-specific target elements in the promoter region of target genes that affect metabolic pathways such as insulin-regulated gluconeogenesis, glucose uptake by muscle cells [41]. Thus, a reduction in PGC-1 $\alpha$ activity is probably caused by the transcriptional effects of Gly482Ser mutation, which might impact the metabolic pathways related to T2DM. To the association of the PGC-1 $\alpha$ polymorphisms with hypertension, it was reported that subjects with (SS) homozygote genotype have lower SBP, which is similar to our findings, and these subjects have a much lower risk of developing hypertension than with (SS) genotype in Danish subjects [42]. Several mechanisms have been proposed for the antihypertensive effects of the PGC-1 $\alpha$. The PGC-1 $\alpha$ loss of function was associated with a reduction in endothelial nitric oxide synthase (eNOS) expression in the endothelium. Conversely, a PGC-1 $\alpha$ gain of function increased basal eNOS expression [43]. The findings of this study suggested that endothelial $P G C-1 \alpha$ protects from vascular dysfunction.

In summary, our findings indicate that $A D I P O Q, L P L$ and $P G C-1 \alpha$ gene polymorphisms can determine genetic susceptibility to MetS as individual biomarkers and their synergistic interaction. On the other hand, genetic studies have provided only limited evidence for a common genetic background of the MetS. Epigenetic factors (DNA methylation and histone modification) are likely to play essential roles in the MetS. Extensive research is needed to clarify the role of genetic variation and epigenetic molecular mechanisms in MetS. We acknowledge the following limitations in the current study. First, selection bias was inevitable; participants of 18-60 age were selected, which might lead to the non-normal distribution of some biochemical parameters. However, we controlled for this by adjusting results with age. Second, our findings are based on a small number of case subjects and need to be replicated in a large population. Third, SNP-environment interactions should also be evaluated to account for MetS risk comprehensively. Nevertheless, our study supports the findings that affecting carbohydrate and lipid metabolism; gene polymorphisms are independent risk factors for the development of MetS.

\section{Conclusions}

Our results suggest that $A D I P O Q+45 \mathrm{~T}>\mathrm{G}, L P L$ PvII and PGC-1 $\alpha$ Gly482Ser loci may contribute to the risk development of MetS in Mongolian subjects.

Author Contributions: Conceptualization, M.J., M.M., and A.C.-E.; methodology, M.J. and A.C.-E.; validation, A.C.-E.; formal analysis, A.C.-E; investigation, A.C.-E., O.S., T.-A.A., P.S., B.D., S.B., L.E., and N.B.; data curation, A.C.-E., B.D., O.S., T.-A.A., P.S., B.D., S.B., L.E., and N.B.; writing-original draft preparation, A.C.-E.; 
writing-review and editing, A.C.-E., M.J., and M.M.; visualization, A.C.-E.; supervision, M.J. and M.M.; project administration, M.J.; funding acquisition, M.J. All authors have read and agreed to the published version of the manuscript.

Funding: This study was supported by a grant (\# 20132211) from the Foundation of Science and Technology, Ministry of Education, Culture, Science and Sports of Mongolia.

Acknowledgments: We would like to thank Curt Chatelain for English editing.

Conflicts of Interest: The authors declare no conflict of interest.

\section{References}

1. Matsuzawa, Y.; Funahashi, T.; Nakamura, T. The concept of metabolic syndrome: Contribution of visceral fat accumulation and its molecular mechanism. J. Atheroscler. Thromb. 2011, 18, 629-639. [CrossRef]

2. Eckel, R.H.; Grundy, S.M.; Zimmet, P.Z. The metabolic syndrome. Lancet 2005, 365, 1415-1428. [CrossRef]

3. Alberti, K.G.; Zimmet, P.Z. Definition diagnosis and classification of diabetes mellitus and its complications. Part 1: Diagnosis and classification of diabetes mellitus provisional report of a WHO consultation. Diabet. Med. 1998, 15, 539-553. [CrossRef]

4. Grundy, S.M.; Brewer, H.B., Jr.; Cleeman, J.I.; Smith, S.C., Jr.; Lenfant, C.; American Heart Association; National Heart, Lung, and Blood Institute. Definition of metabolic syndrome: Report of the National Heart, Lung, and Blood Institute/American Heart Association conference on scientific issues related to definition. Circulation 2004, 109, 433-438. [CrossRef]

5. National Cholesterol Education Program Expert Panel on Detection, Evaluation, and Treatment of High Blood Cholesterol in Adults. Third report of the national cholesterol education program (NCEP) expert panel on detection, evaluation, and treatment of high blood cholesterol in adults (Adult Treatment Panel III) final report. Circulation 2002, 106, 3143-3421. [CrossRef]

6. Grundy, S.M. Metabolic syndrome scientific statement by the American Heart Association and the National Heart, Lung, and Blood Institute. Arter. Thromb. Vasc. Biol. 2005, 25, 2243-2244. [CrossRef]

7. Alberti, K.G.; Zimmet, P.; Shaw, J. Metabolic syndrome-A new world-wide definition. A consensus statement from the international diabetes federation. Diabet. Med. 2006, 23, 469-480. [CrossRef]

8. Enkh-Oyun, T.; Kotani, K.; Davaalkham, D.; Davaa, G.; Ganchimeg, U.; Angarmurun, D.; Khuderchuluun, N.; Batzorig, B.; Tsuboi, B.; Ae, R.; et al. Epidemiologic features of metabolic syndrome in a general Mongolian population. Metab. Syndr. Relat. Disord. 2015, 13, 179-186. [CrossRef]

9. Welty, F.K.; Alfaddagh, A.; Elajami, T.K. Targeting inflammation in metabolic syndrome. Transl. Res. 2016, 167, 257-280. [CrossRef]

10. Magkos, F.; Yannakoulia, M.; Chan, J.L.; Mantzoros, C.S. Management of the metabolic syndrome and type 2 diabetes through lifestyle modification. Ann. Rev. Nutr. 2009, 29, 223-256. [CrossRef]

11. Bozkurt, B.; Aguilar, D.; Deswal, A.; Dunbar, S.B.; Francis, G.S.; Horwich, T.; Jessup, M.; Kosiborod, M.; Pritchett, A.M.; Ramasubbu, K.; et al. Contributory risk and management of comorbidities of hypertension, obesity, diabetes mellitus, hyperlipidemia, and metabolic syndrome in chronic heart failure: A scientific statement from the American Heart Association. Circulation 2016, 134, e535-e578. [CrossRef]

12. Li, P.; Tiwari, H.K.; Lin, W.Y.; Allison, D.B.; Chung, W.K.; Leibel, R.L.; Yi, N.; Liu, N. Genetic association analysis of 30 genes related to obesity in a European American population. Int. J. Obes. 2014, 38, 724-729. [CrossRef]

13. Kathiresan, S.; Willer, C.J.; Peloso, G.M.; Demissie, S.; Musunuru, K.; Schadt, E.E.; Kaplan, L.; Bennett, D.; Li, Y.; Tanaka, T.; et al. Common variants at 30 loci contribute to polygenic dyslipidemia. Nat. Genet. 2009, 41, 56-65. [CrossRef]

14. Marullo, L.; El-Sayed Moustafa, J.S.; Prokopenko, I. Insights into the genetic susceptibility to type 2 diabetes from genome-wide association studies of glycaemic traits. Curr. Diab. Rep. 2014, 14, 551. [CrossRef]

15. International Consortium for Blood Pressure Genome-Wide Association Studies; Ehret, G.B.; Munroe, P.B.; Rice, K.M.; Bochud, M.; Johnson, A.D.; Chasman, D.L.; Smith, A.V.; Tobin, M.V.; Verwoert, G.S.; et al. Genetic variants in novel pathways influence blood pressure and cardiovascular disease risk. Nature 2011, 478, 103-109. 
16. Yamada, Y.; Ichihara, S.; Kato, K.; Yoshida, T.; Yokoi, K.; Matsuo, H.; Watanabe, S.; Metoki, N.; Yoshida, H.; Satoh, K.; et al. Genetic risk for metabolic syndrome: Examination of candidate gene polymorphisms related to lipid metabolism in Japanese people. J. Med. Genet. 2008, 45, 22-28. [CrossRef]

17. Nakayama, K.; Ohashi, J.; Watanabe, K.; Munkhtulga, L.; Iwamoto, S. Evidence for very recent positive selection in Mongolians. Mol. Biol. Evol. 2017, 34, 1936-1946. [CrossRef]

18. Alberti, K.G.; Eckel, R.H.; Grundy, S.M.; Zimmet, P.Z.; Cleeman, J.I.; Donato, K.A.; Fruchart, J.C.; James, W.P.; Loria, C.M.; Smith, S.C. Harmonizing the metabolic syndrome: A joint interim statement of the International Diabetes Federation Task Force on Epidemiology and Prevention; National Heart, Lung, and Blood Institute; American Heart Association; World Heart Federation; International Atherosclerosis Society; and International Association for the Study of Obesity. Circulation 2009, 120, 1640-1645.

19. Suriyaprom, K.; Phonrat, B.; Tungtrongchitr, R. Association of adiponectin gene -11377C >G polymorphism with adiponectin levels and the metabolic syndrome in Thais. Asia. Pac. J. Clin. Nutr. 2014, 23, 167-173.

20. Al-Harithy, R.N.; Al-Zahrani, M.H. The adiponectin gene, ADIPOQ, and genetic susceptibility to colon cancer. Oncol. Lett. 2012, 3, 176-180. [CrossRef]

21. Suriyaprom, K.; Tungtrongchitr, R.; Thawnasom, K. Measurement of the levels of leptin, BDNF associated with polymorphisms LEP G2548A, LEPR Gln223Arg and BDNF Val66Met in Thai with metabolic syndrome. Diabetol. Metab. Syndr. 2014, 6, 6. [CrossRef] [PubMed]

22. Gotoda, T.; Manning, B.S.; Goldstone, A.P.; Imrie, H.; Evans, A.L.; Strosberg, A.D.; McKeigue, P.M.; Scott, J.; Aitman, T.J. Leptin receptor gene variation and obesity: Lack of association in a white British male population. Hum. Mol. Genet. 1997, 6, 869-876. [CrossRef] [PubMed]

23. Al-Jafari, A.A.; Daoud, M.S.; Mobeirek, A.F.; AI Anazi, M.S. DNA polymorphisms of the lipoprotein lipase gene and their association with coronary artery disease in the Saudi population. Int. J. Mol. Sci. 2012, 13, 7559-7574. [CrossRef] [PubMed]

24. Long, X.J.; Yin, R.X.; Li, K.L.; Liu, W.Y.; Zhang, L.; Cao, X.L.; Miao, L.; Wu, D.F.; Htet Aung, L.H.; Hu, X.J.; et al. Low density lipoprotein receptor gene Ava II polymorphism and serum lipid levels in the Guangxi Bai $\mathrm{Ku}$ Yao and Han populations. Lipids. Health. Dis. 2011, 10, 34. [CrossRef]

25. Fu, Y.; Katsuya, T.; Higaki, J.; Asai, T.; Fukuda, M.; Takiuchi, S.; Hatanaka, Y.; Rakugi, H.; Ogihara, T. A common mutation of low-density lipoprotein receptor gene is associated with essential hypertension among Japanese. J. Hum. Hypertens. 2001, 15, 125-130. [CrossRef]

26. Ridderstrale, M.; Johansson, L.E.; Rastam, L.; Lindblad, U. Increased risk of obesity associated with the variant allele of the PPARGC1A Gly482Ser polymorphism in physically inactive elderly men. Diabetologia 2006, 49, 496-500. [CrossRef]

27. Kraja, A.T.; Vaidya, D.; Pankow, J.S.; Goodarzi, M.O.; Assimes, T.L.; Kullo, I.J.; Sovio, U.; Mathias, R.A.; Sun, Y.V.; Franceschini, N.; et al. A bivariate genome-wide approach to metabolic syndrome: STAMPEED consortium. Diabetes 2011, 60, 1329-1339. [CrossRef]

28. Kristiansson, K.; Perola, M.; Tikkanen, E.; Kettunen, J.; Surakka, I.; Havulinna, A.S.; Stančáková, A.; Barnes, C.; Widen, E.; Kajantie, E.; et al. Genome-wide screen for metabolic syndrome susceptibility Loci reveals strong lipid gene contribution but no evidence for common genetic basis for clustering of metabolic syndrome traits. Circ. Cardiovasc. Genet. 2012, 5, 242-249. [CrossRef]

29. Delgado-Lista, J.; Perez-Martinez, P.; Solivera, J.; Garcia-Rios, A.; Perez-Caballero, A.I.; Lovegrove, J.A.; Drevon, C.A.; Defoort, C.; Blaak, E.E.; Dembinska-Kieć, A.; et al. Top single nucleotide polymorphisms affecting carbohydrate metabolism in metabolic syndrome: From the LIPGENE study. J. Clin. Endocrinol. Metab. 2014, 99, e384-e389. [CrossRef]

30. Tekola-Ayele, F.; Doumatey, A.P.; Shriner, D.; Bentley, A.R.; Chen, G.; Zhou, J.; Fasanmade, O.; Johnson, T.; Oli, J.; Okafor, G.; et al. Genome-wide association study identifies African-ancestry specific variants for metabolic syndrome. Mol. Genet. Metab. 2015, 116, 305-313. [CrossRef]

31. Gao, M.; Ding, D.; Huang, J.; Qu, Y.; Wang, Y.; Huang, Q. Association of genetic variants in the adiponectin gene with metabolic syndrome: A case-control study and a systematic meta-analysis in the Chinese population. PLoS ONE 2013, 8, e58412. [CrossRef] [PubMed]

32. Woo, J.G.; Dolan, L.M.; Deka, R.; KaushaI, R.D.; Shen, Y.; Pal, P.; Daniels, S.R.; Martin, L.J. Interactions between noncontiguous haplotypes in the adiponectin gene ACDC are associated with plasma adiponectin. Diabetes 2006, 55, 523-529. [CrossRef] [PubMed] 
33. Zhao, T.; Zhao, J. Genetic effects of adiponectin on blood lipids and blood pressure. Clin. Endocrinol. 2011, 74, 214-222. [CrossRef] [PubMed]

34. Nam, J.S.; Han, J.W.; Lee, S.B.; You, J.H.; Kim, M.J.; Kang, S.; Park, J.S.; Ahn, C.W. Calpain-10 and adiponectin gene polymorphisms in Korean type 2 diabetes patients. Endocrinol. Metab. 2018, 33, 364-371. [CrossRef] [PubMed]

35. Wang, C.; Sun, T.; Li, H.; Ba, J.; Li, Y. Lipoprotein lipase Ser447Ter polymorphism associated with the risk of ischemic stroke: A meta-analysis. Thromb. Res. 2011, 128, e107-e112. [CrossRef]

36. Bozina, T.; Simic, I.; Lovric, J.; Pecin, I.; Jelakovic, B.; Sertic, J.; Reiner, Z. Effects of lipoprotein lipase and peroxisome proliferator-activated receptor-gamma gene variants on metabolic syndrome traits. Coll. Antropol. 2013, 37, 801-808.

37. Kim, Y.; Lee, M.; Lim, Y.; Jang, Y.; Park, H.K.; Lee, Y. The gene-diet interaction, LPL PvuII and HindIII and carbohydrate, on the criteria of metabolic syndrome: KMSRI-Seoul Study. Nutrition 2013, 29, 1115-1121. [CrossRef]

38. Banks, W.A.; Farr, S.A.; Salameh, T.S.; Niehoff, M.L.; Rhea, E.M.; Morley, J.E.; Hanson, A.J.; Hansen, K.M.; Craft, S. Triglyceride cross the blood-barrier and induce central leptin and insulin receptor resistance. Int. J. Obes. 2018, 42, 391-397. [CrossRef]

39. Ambye, L.; Rasmussen, S.; Fenger, M.; Jorgensen, T.; Borch-Johnsen, K.; Madsbad, S.; Urhammer, S.A. Studies of the Gly482Ser polymorphism of the peroxisome proliferator-activated receptor $\gamma$ coactivator $1 \alpha($ PGC- $1 \alpha)$ gene in Danish subjects with the metabolic syndrome. Diabetes. Res. Clin. Pr. 2005, 67, 175-179. [CrossRef]

40. Hara, K.; Tobe, K.; Okada, T.; Kadowaki, H.; Akanuma, Y.; Ito, C.; Kimura, S.; Kadowaki, T. A genetic variation in the PGC-1 gene could confer insulin resistance and susceptibility to Type II diabetes. Diabetology 2002, 45, 740-743. [CrossRef]

41. Michael, L.F.; Wu, Z.; Cheatham, R.B.; Puigserver, P.; Adelmant, G.; Lehman, J.J.; Kelly, D.P.; Spiegelman, B.M. Restoration of insulin-sensitive glucose transporter (GLUT4) gene expression in muscle cells by the transcriptional coactivator PGC-1. Proc. Natl. Acad. Sci. USA 2001, 98, 3820-3825. [CrossRef]

42. Andersen, G.; Wegner, L.; Jensen, D.P.; Glümer, C.; Tarnow, L.; Drivsholm, T.; Poulsen, P.; Hansen, S.K.; Nielsen, E.D.; Ek, K.; et al. PGC-1 $\alpha$ Gly482Ser polymorphism associates with hypertension among Danish whites. Hypertension 2005, 45, 565-570. [CrossRef]

43. Craige, S.M.; Kröller-Schön, S.; Li, C.; Kant, S.; Cai, S.; Chen, K.; Contractor, M.M.; Pei, Y.; Schulz, E.; Keaney, J.F., Jr. PGC-1 $\alpha$ dictates endothelial function through regulation of eNOS expression. Sci. Rep. 2016, 6, 38210. [CrossRef] [PubMed] 